\title{
Relating the cosmological constant and supersymmetry breaking in warped compactifications of IIB string theory
}

\author{
P. Berglund* \\ CIT-USC Center for Theoretical Physics, Department of Physics and Astronomy, University of Southern California, \\ Los Angeles, California 90089-0484 \\ T. Hübsch ${ }^{\dagger}$ \\ Department of Physics and Astronomy, Howard University, Washington, D.C. 20059 \\ D. Minic \\ Institute for Particle Physics and Astrophysics, Department of Physics, Virginia Tech, Blacksburg, Virginia 24061
}

(Received 13 March 2002; revised manuscript received 29 October 2002; published 27 February 2003)

\begin{abstract}
It has been suggested that the observed value of the cosmological constant is related to the supersymmetry breaking scale $M_{\text {susy }}$ through the formula $\Lambda \sim M_{p}^{4}\left(M_{\text {susy }} / M_{p}\right)^{8}$. We point out that a similar relation naturally arises in the codimension two solutions of warped spacetime varying compactifications of string theory, in which nonisotropic stringy moduli induce a small but positive cosmological constant.
\end{abstract}

DOI: 10.1103/PhysRevD.67.041901

PACS number(s): 11.25.Mj, 11.10.Kk, 98.80.Cq

Recently, we addressed the question of whether de Sitter space [1] can be obtained from string theory [2]. Such nonsingular, nonstatic spacetimes fall into the class of codimension two nonsupersymmetric string vacua studied in Refs. [3, $4,5] .{ }^{1}$ In these models, supersymmetry is explicitly broken by a global cosmic brane [6] with a core of size $\ell$, extended along the $D-2$ "longitudinal" directions. While Refs. [3, 4, 5] considered a flat Minkowski brane, the main point of Ref. [2] is the existence of nonsupersymmetric codimension two solutions with a positive cosmological constant, $\Lambda_{b}$, in the $(D-2)$-dimensional longitudinal space. ${ }^{2}$

Since the cosmological constant in our model [2] is directly related to the nonisotropy of matter, we may compare it with various attempts to incorporate Mach's principle in string theory $[8,9]$ as well with the idea that supersymmetry breaking might have a cosmological origin $[10,11]$. In particular, it has been suggested that the observed value of the cosmological constant, $\Lambda_{4} \sim 10^{-44} \mathrm{GeV}^{4}[12,13,7]$, may be related to the supersymmetry breaking scale $M_{\text {susy }}$ through the formula $[14,15,10]$

$$
\Lambda_{4} \sim M_{p}^{4}\left(M_{\text {susy }} / M_{p}\right)^{8}
$$

with $M_{\text {susy }} \sim 10 \mathrm{TeV}$ and $M_{p} \sim 10^{19} \mathrm{GeV}$ as appropriate in

\footnotetext{
*Email address: berglund@citusc.usc.edu

†On leave from the "Rudjer Bošković" Institute, Zagreb, Croatia. Email address: thubsch@howard.edu

*Email address: dminic@vt.edu

${ }^{1}$ By string vacua we denote solutions that satisfy the corresponding type IIB supergravity equations of motion and contain moduli with proper $\operatorname{SL}(2, Z)$ properties.

${ }^{2}$ Note that $\Lambda_{b}>0$ removes the naked singularity present in the model considered in $[3,4,5]$, in comparison with earlier discussions of a positive cosmological constant along the brane world [7].
}

four dimensions. ${ }^{3}$ It is the aim of this work to point out that the setup of Ref. [2] for $D=6$ leads naturally to a relation analogous to Eq. (1). In particular, the stringy moduli induce a nontrivial relation between the scale of the global cosmic brane, $\ell$, the nonisotropy of matter, $\omega$, induced by the brane, and $\Lambda_{b}$ [2]. We take this observation one step further and find an explicit relation between $\Lambda_{b}, \ell$, and the natural mass scales in this theory, $M_{6}$ and $M_{4}$-the Planck scales in the bulk and along the brane, respectively, thus deriving an equation analogous to Eq. (1).

Although the detailed physics leading to this relation is unclear, we find it very intriguing that an equation similar to Eq. (1) emerges naturally in our framework. Still, one of the most important unresolved questions in the scenario presented in Ref. [2] is the issue of the stability of this nonsupersymmetric background in the full string theory. Since supersymmetry is broken, one also has to address the effects of stringy corrections. We will argue that those corrections are negligible.

The general framework of our analysis is as in Refs. [3, 4, $5,2]$, to which we refer the reader for a more detailed analysis. Although we will be mostly interested in the phenomenologically relevant case in which $D=6$, and hence the uncompactified spacetime is $D-2=$ four dimensional, we find it useful to work in a general $D$-dimensional background. We consider type IIB string theory (compactified on a fixed su-

\footnotetext{
${ }^{3}$ The essential ingredient of this proposal is a conjectured relevance of the nondecoupling between the microscopic and macroscopic degrees of freedom $[9,10,13,7]$ for the cosmological constant problem. This conjecture is natural from the following intuitive perspective on the cosmological constant problem. On one hand, the cosmological constant is tied to the fundamental physics of the vacuum, because $\Lambda_{4}$ is essentially given by the vacuum energy density. On the other hand, the cosmological constant is related to the large scale behavior of the universe, since a small cosmological constant implies that the observable universe is big and almost flat.
} 
persymmetry preserving space) in which the axion-dilaton system $(\alpha, \phi)$, described by the complex modulus field $\tau$ $=\alpha+i \exp (-\phi)$, varies over the $x_{D-2, D-1}$ directions of the uncompactified spacetime. Thus, the relevant part of the lowenergy effective $D$-dimensional action of the modulus, $\tau$, coupled to gravity reads

$$
S_{\mathrm{eff}}=\frac{1}{2 \kappa^{2}} \int d^{D} x \sqrt{-g}\left(R-\mathcal{G}_{\tau \bar{\tau}} g^{\mu \nu} \partial_{\mu} \tau \partial_{\nu} \bar{\tau}+\cdots\right)
$$

Here $\mu, \nu=0, \ldots, D-1,2 \kappa^{2}=16 \pi G_{N}^{(D)}$, where $G_{N}^{(D)}$ is the $D$-dimensional Newton constant, and $\mathcal{G}_{\tau \bar{\tau}}=-(\tau-\bar{\tau})^{-2}$ is the metric on the complex structure moduli space of a torus. ${ }^{4}$

Let us now briefly review the codimension two solution with positive cosmological constant $\Lambda_{b}$ along the longitudinal direction of the cosmic brane [2]. The metric ansatz is

$$
\begin{array}{r}
\mathrm{d} s^{2}=A^{2}(z) \bar{g}_{a b} \mathrm{~d} x^{a} \mathrm{~d} x^{b}+\ell B^{2}(z)\left(\mathrm{d} z^{2}+\mathrm{d} \theta^{2}\right), \\
\bar{g}_{a b} \mathrm{~d} x^{a} \mathrm{~d} x^{b}=-\mathrm{d} x_{0}^{2}+e^{2 \sqrt{\lambda_{b}} x_{0}}\left(\mathrm{~d} x_{1}^{2}+\cdots+\mathrm{d} x_{D-3}^{2}\right),
\end{array}
$$

where $z=\log (r / \ell)$. As in the case when $\Lambda_{b}=0$, we find that the explicit solutions for $\tau$ are aperiodic, such as $\tau=\alpha_{0}$ $+i g_{s}^{-1} \exp (\omega \theta)$, but do exhibit a nontrivial SL $(2, \mathbb{Z})$ monodromy [3]. ${ }^{5}$ This ensures that our solution is stringy (although classical and nonsupersymmetric) rather than merely a supergravity vacuum. Note, in particular, that the dilaton of the type IIB superstring theory varies with the polar angle, not the radial distance. Recall that with $\tau=\alpha_{0}$ $+i g_{s}^{-1} \exp (\omega \theta)$, the $\operatorname{SL}(2, Z)$ symmetry requires $g_{s}^{D} \sim O(1)$ in $D$ dimensions. However, in the $(D-2)$-dimensional brane world, $g_{s}^{D-2}=g_{s}^{D} \sqrt{\alpha^{\prime} / V_{\perp}}$, and since $V_{\perp}$, the volume of the transversal space, is large [3], $g_{s}^{D-2} \ll 1$. Below, we will return to discussing the corrections to our classical solution.

Following [2], the Einstein equation can be simplified,

$$
R_{\mu \nu}=\mathcal{G}_{\tau \bar{\tau}} \partial_{\mu} \tau \partial_{\nu}{ }_{\nu} \bar{\tau}=\widetilde{T}_{\mu \nu}
$$

Since the metric (3) is axially symmetric, while $\tau$ is independent of the radial distance from the cosmic brane, $\widetilde{T}_{\mu \nu}$ $=\operatorname{diag}\left[0, \ldots, 0, \frac{1}{4} \omega^{2} \ell^{-2}\right]$. Equation (5) then defines the general class of our spacetimes as almost Ricci flat: $R_{\mu \nu}$ $=\operatorname{diag}\left[0, \ldots, 0, \frac{1}{4} \omega^{2} \ell^{-2}\right]$, where $\omega^{2}>0$ is indeed related to supersymmetry breaking [3] and $\ell$ is the (transversal) length scale of the cosmic brane.

The $R_{a b}=0$ part of Eq. (5) reduces to a single equation, giving

\footnotetext{
${ }^{4}$ Recall that because of its $\operatorname{SL}(2, \mathrm{Z})$ properties, the axion-dilaton, $\tau$, can be thought of as the complex structure of a $T^{2}$, in analogy with $F$ theory [16].

${ }^{5}$ Although $\partial \tau$ does not transform correctly under $\operatorname{SL}(2, \mathbb{Z})$ transformations, it is straightforward to show that $\mathcal{G}_{\tau \bar{\tau}}^{-1}|\partial \tau|^{2}$, which appears in the action (2), is invariant.
}

$$
\begin{aligned}
B^{2} & =\ell^{-2} \Lambda_{b}^{-1}\left(A^{\prime 2}+\frac{1}{(D-3)} A A^{\prime \prime}\right) \\
& =\ell^{-2} \Lambda_{b}^{-1} \frac{h^{\prime \prime} h^{-(D-4) /(D-2)}}{(D-2)(D-3)},
\end{aligned}
$$

which determines $B(z)$ in terms of $A(z)$ or $h(z)$ def $=A(z)^{D-2}$. With this substitution, the remaining components of Eq. (5) reduce to the following equation:

$$
\frac{1}{2(D-2)} \frac{h^{\prime 2}}{h^{2}}-\frac{h^{\prime \prime}}{2 h}+\frac{h^{\prime} h^{\prime \prime \prime}}{2 h h^{\prime \prime}}=-\frac{1}{8} \omega^{2} \text {. }
$$

For $\omega \neq 0(\tau \neq$ const $)$, Eq. (7) has a perturbative, analytic solution: ${ }^{6}$

$$
\begin{aligned}
& A(z)=Z(z)\left(1-\frac{\omega^{2} \rho_{0}^{2}(D-3)}{24(D-1)(D-2)} Z(z)^{2}+O\left(\omega^{4}\right)\right), \\
& B(z)=\frac{1}{\ell \rho_{0} \sqrt{\Lambda_{b}}}\left(1-\frac{\omega^{2} \rho_{0}^{2}}{8(D-1)} Z(z)^{2}+O\left(\omega^{4}\right)\right),
\end{aligned}
$$

where $Z(z)=1-z / \rho_{0}$ and $\rho_{0}>0$. As was shown in Ref. [2], close to the horizon, spacetime is asymptotically flat in agreement with the behavior of Rindler space [18]. $[6,3]$

In contrast, when $\Lambda_{b}=0$, the solution is very different

$$
\begin{aligned}
& \widetilde{A}(z)=\widetilde{Z}(z)^{1 /(D-2)}, \\
& \widetilde{B}(z)=\widetilde{Z}(z)^{-(D-3) / 2(D-2)} e^{\left(\xi / 2 a_{0}\right)\left[1-\tilde{Z}(z)^{2}\right]},
\end{aligned}
$$

where now $\widetilde{Z}=1-a_{0} z$, and we restrict to $a_{0}>0$. This solution exhibits a naked singularity, at $z=a_{0}^{-1}(\widetilde{Z}=0)$, for the global cosmic brane.

While the naked singularity has been removed by $\Lambda_{b}$ $>0$, it was first shown by Gregory [17] and by [2] that the global cosmic brane solution (9) is still a good approximation to Eq. (8) away from the horizon. In particular, by comparing Eq. (8) with Eq. (9) close to the core, one can show that

$$
\begin{gathered}
a_{0}=-\left.\frac{h^{\prime}}{h}\right|_{z=0}, \quad \xi=\left.\left(\frac{h^{\prime \prime}}{2 h^{\prime}}-\frac{\omega^{2} h}{8 h^{\prime}}\right)\right|_{z=0}, \\
\ell=\Lambda_{b}^{-1 / 2}\left(\left.\frac{h^{\prime \prime} h^{-(D-4) /(D-2)}}{(D-2)(D-3)}\right|_{z=0}\right)^{1 / 2} .
\end{gathered}
$$

That is, given a smooth solution defined by Eq. (8) and parameterized in terms of $\left(\rho_{0}, \omega, \Lambda_{b}\right)$, this solution close to $z$ $=0$ can be interpreted as a global cosmic brane solution with parameters $\left(a_{0}, \xi, \ell\right)$ determined by $\left(\rho_{0}, \omega, \Lambda_{b}\right)$ through Eqs. (10) and (11). Alternatively, we can solve for $\Lambda_{b}$,

\footnotetext{
${ }^{6}$ This solution is of the same form as that discussed by Gregory [17] for the U(1) vortex solution.
} 


$$
\Lambda_{b}=\frac{\left.\left(\omega^{2}-\omega_{\mathrm{GCB}}^{2}\right) A^{2}\right|_{z=0}}{4 \ell^{2}(D-2)(D-3)},
$$

where $\omega_{\mathrm{GCB}}^{2}=8 a_{0} \xi[3]$. Note that $\omega_{\mathrm{GCB}}^{2}$ comes from the stress tensor associated with the global cosmic brane to which the solution tends asymptotically when $z \rightarrow 0$, while $\omega^{2}$ is the stress tensor for the $\Lambda_{b}>0$ solution. Thus, the cosmological constant is directly related to the nontrivial variation of the matter as a function of $\theta$. This gives a very nontrivial relation between the stringy moduli, and hence string theory itself, and a positive $\Lambda_{b}$. Furthermore, $\Lambda_{b}>0$ implies that ${ }^{7} \omega^{2}>\omega_{\mathrm{GCB}}^{2}$. When $\omega^{2}=0$ it then follows that $\omega_{\mathrm{GCB}}^{2}=0$. The latter is a necessary condition for obtaining a supersymmetric configuration. Thus, we see the important relation between supersymmetry breaking and a positive cosmological constant.

Finally, the Newton constant $G_{N}^{(D-2)}=M_{D-2}^{-(D-4)}$ in $D$ -2 dimensions and the zero-mode wave function normalization $\left\langle\psi_{0} \mid \psi_{0}\right\rangle$ are [2]

$$
\begin{gathered}
G_{N}^{(D-2)}=M_{D}^{-(D-2)}\left\langle\psi_{0} \mid \psi_{0}\right\rangle^{-1}, \\
\left\langle\psi_{0} \mid \psi_{0}\right\rangle \sim \frac{\pi}{D-3} \frac{\ell}{\sqrt{\Lambda_{b}}} .
\end{gathered}
$$

The volume of the transversal space $V_{\perp}=\left\langle\psi_{0} \mid \psi_{0}\right\rangle$ is large [2] and drives the large $M_{D-2} / M_{D}$ hierarchy. This then implies the following relation:

$$
\Lambda_{D-2} \sim\left(\frac{\pi}{D-3}\right)^{2} M_{D-2}^{D-2}\left(\ell M_{D-2}\right)^{2}\left(\frac{M_{D}}{M_{D-2}}\right)^{2 D-4},
$$

where $\Lambda_{D-2}=\Lambda_{b} / G_{N}^{(D-2)}$ is the energy density in $D-2$ dimensions.

From now on we will focus on the phenomenologically relevant case of $D=6$. Recall that $\ell$ is the characteristic (transverse) size of the cosmic brane, for the formation of which no concrete physical mechanism is known. However, should $\ell$ be stabilized by a longitudinal four-dimensional physical mechanism, ${ }^{8}$ then $\ell \sim M_{4}^{-1}$ and [up to factors of $O(1)]$

$$
\Lambda_{4} \sim M_{4}^{4}\left(\frac{M_{6}}{M_{4}}\right)^{8} .
$$

The original scenario of Ref. [2] then applies, where the ten-dimensional spacetime of the type IIB string theory is compactified on a four-dimensional supersymmetry preserving space $^{9}$ of characteristic size $M_{10}^{-1}=M_{6}^{-1} \sim(10 \mathrm{TeV})^{-1}$

\footnotetext{
${ }^{7}$ That $\Lambda_{b}$ is indeed positive can be seen from Eq. (6). At the horizon, $A\left(z=\rho_{0}\right)=0$, which implies that the right hand side of Eq. (6) is positive if $\Lambda_{b}>0$.

${ }^{8}$ There exist both field and string theory arguments of this type [19].

${ }^{9}$ All remaining supersymmetry will be broken by the cosmic brane solution [3].
}

$\sim 10^{-19} \mathrm{~m}$. The cosmic brane of Ref. [2] then describes a $(3+1)$-dimensional de Sitter world brane, with the characteristic scale $M_{4} \sim 10^{19} \mathrm{GeV}$. Furthermore, $L=\Lambda_{b}^{-1 / 2}$ $\sim 10^{41} \mathrm{GeV}^{-1} \sim 10^{25} \mathrm{~m}$ provides a natural scale that coincides with the Hubble radius.

Note that Eq. (15) is an equation of the same form as the desired relationship (1) upon identifying $M_{10}=M_{6}$ with the scale of supersymmetry breaking, and $M_{4}$ with the fourdimensional Planck scale $M_{p}$. More precisely, Eq. (15) provides an explicit relation between the value of the cosmological constant and the hierarchy involving the two fundamental scales. Without a detailed dynamical mechanism, it is of course very difficult to argue that $M_{6}$ should be precisely identified with the scale of supersymmetry breaking. Nevertheless, as we will indicate in the concluding paragraph, the idea that the cosmological constant and supersymmetry breaking are related is natural in our model. As far as we know, this is the first time such a relation between the observed value of the cosmological constant and the scale of supersymmetry breaking has been obtained in a specific dynamical situation. Note that this relation crucially depends on the fact that the zero-mode normalization scales as $\left\langle\psi_{0} \mid \psi_{0}\right\rangle$ $\sim \ell / \sqrt{\Lambda_{b}}$, which is a specific feature of the scenario presented in [2].

In fact, there exists a whole spectrum of scenarios, albeit with powers of the mass scale ratio in Eq. (14) which may not be 8 as in Eq. (15). These scenarios differ in the compactification/cosmic brane ansatz sequencing. For example, let the ten-dimensional spacetime of the type IIB string theory first be compactified on a three-dimensional supersymmetry preserving space of characteristic size $M_{10}^{-1}$ $=M_{7}^{-1} \sim(10 \mathrm{TeV})^{-1} \sim 10^{-20} \mathrm{~m}$. Assuming that $\ell$ is stabilized by the "bulk" seven-dimensional physics, then $\ell$ $\sim\left(M_{7}\right)^{-1}$ and $\Lambda_{5} \sim M_{5}^{5}\left(M_{7} / M_{5}\right)^{8}$. Upon a Kaluza-Klein compactification on a circle of radius $M_{5}^{-1} \sim\left(10^{19} \mathrm{GeV}\right)^{-1}$ $\sim 10^{-35} \mathrm{~m}$, this yields a $(3+1)$-dimensional de Sitter world brane with $M_{4}=M_{5} \sim 10^{19} \mathrm{GeV}$. On the other hand, for a codimension two cosmic brane in ten dimensions with $\ell$ stabilized by the longitudinal eight-dimensional physics, $\Lambda_{8}$ $\sim M_{8}^{8}\left(M_{10} / M_{8}\right)^{16}$. After wrapping on a suitable fourdimensional space (of size $M_{8}^{-1}$ ), for the desirable values of $\Lambda_{4} \sim 10^{-44} \mathrm{GeV}^{4}$ and $M_{4}=M_{8} \sim 10^{19} \mathrm{GeV}$, we find that $M_{10} \sim 5.6 \times 10^{6} \mathrm{GeV}$ is the fundamental scale. At the opposite end, by compactifying the ten-dimensional spacetime on a suitable four-dimensional space and then constructing a codimension two cosmic brane in six dimensions with $\ell$ stabilized by the bulk six-dimensional physics, $\Lambda_{4}$ $\sim M_{4}^{4}\left(M_{6} / M_{4}\right)^{6}$. For the desirable values of $\Lambda_{4}$ $\sim 10^{-44} \mathrm{GeV}^{4}$ and $M_{8}=M_{4} \sim 10^{19} \mathrm{GeV}$, the fundamental scale becomes $M_{6}=M_{10} \sim 100 \mathrm{MeV}$.

Finally, let us conclude by discussing the stringy and quantum corrections to our solution. We will assume that the six-dimensional theory has the equivalent of $N=4$ supersymmetry in four dimensions, or equivalently 16 supercharges. This will always be the case as long as we are considering type II theories with at most a K3 compactification from ten to six dimensions. First, note that $\left.\Lambda_{b} \sim h^{\prime \prime}\right|_{z=0} \ell^{-2}$ 
[which follows from Eq. (11)] is consistent with the notion that supersymmetry breaking and a nonzero cosmological constant are related. To see this, first recall that, from Ref. [4], the supersymmetry breaking is indicated by the nonvanishing of $d A / d r$. But $A^{\prime} \sim A h^{\prime} / h$, so supersymmetry is broken when $h^{\prime} \neq 0$, which in turn implies that $h^{\prime \prime} \neq 0$ and hence ${ }^{10} \Lambda_{b}>0$. Furthermore, from $h=A^{D-2}$ it follows that (at least close to the horizon) $A^{\prime} \sim\left(h^{\prime \prime} h^{-(D-4) /(D-2)}\right)^{1 / 2}$ $\sim \Lambda_{b}^{1 / 2} \ell^{-1}$. With $\ell \sim M_{4}^{-1}=10^{-19} \mathrm{GeV}^{-1}$ and $\Lambda_{b}$ $\sim 10^{-82} \mathrm{GeV}^{2}$, we find $A^{\prime} \sim 10^{-60}$, which is a very small number. This, we argue, justifies neglecting the corrections due to supersymmetry breaking. ${ }^{11}$ The $\alpha^{\prime}$ corrections due to the global cosmic string would have to take the form $\alpha^{\prime} / V_{\perp}$, where $\alpha^{\prime} \sim M_{6}^{-2}$ is the string scale. From Eq. (13) it then follows that the string corrections are very small. Now, al-

\footnotetext{
${ }^{10}$ If $h^{\prime} \neq 0$ and $h^{\prime \prime}=0$, then this describes the singular global cosmic brane solution in which $\Lambda_{b}=0$.

${ }^{11}$ In general, there will be $\alpha^{\prime}$ and string coupling corrections without breaking supersymmetry; these will not be considered here.
}

though our solution is not the Bogomol'nyi-PrasadSommerfield solution, supersymmetry is broken very weakly. Therefore, the corrections should be proportional both to the coupling and the supersymmetry breaking parameter. Since the six-dimensional string coupling $g_{s}^{6} \sim O(1)$ because of modular invariance, the four-dimensional string coupling $g_{s}^{4}$ $\ll 1$ as discussed above. Then, the smallness of the supersymmetry breaking parameter justifies neglecting strong coupling corrections.

We are indebted to V. Balasubramanian, J. de Boer, S. Kachru, N. Kaloper, and F. Larsen for very useful discussions. P.B. would like to thank the organizers of the Lake Arrowhead workshop on "New Directions in Conformal Field Theory" for a stimulating environment, as well as CIG, Berkeley. T.H. wishes to thank the Caltech-USC Center for Theoretical Physics for its hospitality. The work of P.B. and T.H. was supported by the U.S. Department of Energy under grants Nos. DE-FG03-84ER40168 and DE-FG02-94ER40854, respectively. D.M. would like to thank the organizers of the KIAS Winter School in String Theory for hospitality and for providing a stimulating working environment.
[1] For recent reviews and references, consult, for example, V. Balasubramanian, P. Horava, and D. Minic, J. High Energy Phys. 05, 043 (2001); E. Witten, hep-th/0106109; A. Strominger, J. High Energy Phys. 10, 034 (2001); M. Spradlin, A. Strominger, and A. Volovich, hep-th/0110007; C. M. Hull, J. High Energy Phys. 11, 012 (2001); V. Balasubramanian, J. de Boer, and D. Minic, Phys. Rev. D 65, 123508 (2002); R. Bousso, A. Maloney, and A. Strominger, ibid. 65, 104039 (2002).

[2] P. Berglund, T. Hübsch, and D. Minic, Phys. Lett. B 534, 147 (2002).

[3] P. Berglund, T. Hübsch, and D. Minic, J. High Energy Phys. 09, 015 (2000); 02, 010 (2001).

[4] P. Berglund, T. Hübsch, and D. Minic, J. High Energy Phys. 01, 041 (2001).

[5] P. Berglund, T. Hübsch, and D. Minic, Phys. Lett. B 612, 155 (2001).

[6] A.G. Cohen and D.B. Kaplan, Phys. Lett. B 470, 52 (1999); 215, 663 (1988).

[7] S. Kachru, M. Schulz, and E. Silverstein, Phys. Rev. D 62, 045021 (2000); N. Arkani-Hamed, S. Dimopoulos, N. Kaloper, and R. Sundrum, Phys. Lett. B 480, 193 (2000).

[8] P. Horava, Phys. Rev. D 59, 046004 (1999); P.K. Townsend, hep-th/9903043.
[9] P. Horava and D. Minic, Phys. Rev. Lett. 85, 1610 (2000).

[10] T. Banks, Int. J. Mod. Phys. A 16, 910 (2001); hep-th/0007146.

[11] B.B. Deo and S.J. Gates, Phys. Lett. 151B, 195 (1985).

[12] For observational constraints on the cosmological constant and theoretical approaches to it, see S.M. Carroll, Living Rev. Relativ. 4, 1 (2001). For some theoretical perspectives, see S. Weinberg, astro-ph/0005265; Rev. Mod. Phys. 61, 1 (1989); E. Witten, hep-ph/0002297; J. Polchinski, String Theory (Cambridge University Press, Cambridge, 1998).

[13] For other related attempts to understand the cosmological constant problem, consult, for example, T. Banks, hep-th/9601151; A.G. Cohen, D.B. Kaplan, and A.E. Nelson, Phys. Rev. Lett. 82, 4971 (1999); L. N. Chang, D. Minic, N. Okamura, and T. Takeuchi, Phys. Rev. D 65, 125028 (2002); 65, 125027 (2002).

[14] E. Kiritsis, J. High Energy Phys. 10, 010 (1999).

[15] J.E. Kim, J. High Energy Phys. 06, 016 (2000).

[16] C. Vafa, Nucl. Phys. B469, 403 (1996).

[17] R. Gregory, Phys. Rev. Lett. 84, 2564 (2000); Phys. Rev. D 54, 4955 (1996).

[18] N. Kaloper, Phys. Rev. D 60, 123506 (1999).

[19] I. Antoniadis, R. Minasian, and P. Vanhove, Nucl. Phys. B648, 69 (2003); G. R. Dvali and G. Gabadadze, Phys. Rev. D 63, 065007 (2001); E. Kiritsis, N. Tetradis, and T. N. Tomaras, J. High Energy Phys. 08, 012 (2001). 